\title{
二芳基碘鎓盐在有机反应中的研究新进展
}

\author{
肖志超 $a, b$ 夏成峰 $*, a$ \\ ( ${ }^{a}$ 中国科学院昆明植物研究所 植物化学与西部植物资源持续利用国家重点实验室 昆明 650201) \\ $\left({ }^{b}\right.$ 中国科学院大学 北京 100039)
}

\begin{abstract}
摘要 二芳基碘鎓盐作为一种温和、无毒且性质稳定的芳基化试剂, 近年来在有机反应中得到广泛的应用. 综述了它 的结构和活性特点、制备方法、参与的 $\mathrm{C}-\mathrm{H}$ 芳基化反应、羰基化合物的芳基化反应、串联环化反应以及对杂原子亲 核试剂的芳基化反应的研究进展，并阐述了各类反应的特点、机理和应用.

关键词 二芳基碘鉾盐; 芳基化; 偶联反应
\end{abstract}

\section{Progresses of Diaryliodonium Salts in Organic Reactions}

\author{
Xiao, Zhichao ${ }^{a, b} \quad$ Xia, Chengfeng ${ }^{*, a}$ \\ ( ${ }^{a}$ State Key Laboratory of Phytochemistry and Plant Resources in West China, Kunming Institute of Botany, \\ Chinese Academy of Sciences, Kunming 650201) \\ ( ${ }^{b}$ University of Chinese Academy of Sciences, Beijing 100039)
}

\begin{abstract}
Diaryliodonium salts, a kind of mild, non-toxic and stable arylation reagent, have wide applications in organic chemistry. This review presents its structure and reaction activity characteristics, preparation methods, recent progresses in arylation of $\mathrm{C}-\mathrm{H}$ bond, arylation of carbonyl compounds, cascade reaction, as well as arylation of hetero-atom nucleophiles. The characteristics of the reaction mechanisms and their applications are also discussed.

Keywords diaryliodonium salt; arylation; cross-coupling reaction
\end{abstract}

高价碘化学的历史可以追溯到 1886 年, 德国化学 家 Willgerodt ${ }^{[1]}$ 在冰浴条件下向碘苯溶液中通入氯气, 溶液中析出针状黄色晶体, 这黄色晶体就是(二氯碘)苯 1. Willgerodt ${ }^{[2]}$ 将(二氯碘)-苯在醋酸条件下水解, 得 到碘苯二乙酸 2. 后来, Hartmann 和 Meyer 合作相继合 成出 $3(\mathrm{IBX})^{[3]}$ 和二苯基碘鎓盐 $\mathbf{4}^{[4]}$. 这一系列重要的高 价碘试剂奠定了今天高价碘化学 ${ }^{[5]}$ 的基础(图 1).

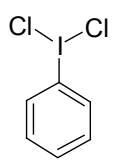

1

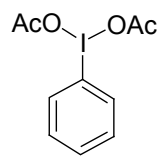

2<smiles>O=C1OI(=O)(O)c2ccccc21</smiles>

3 (IBX)

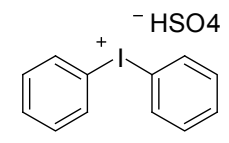

4
图 1 早期发现的重要高价碘试剂

Figure 1 Earlier developed important hypervalent iodine reagents
二芳基碘鎓盐是高价碘化学的一个重要组成部分, 但它在 1894 年被合成以后一直没有得到应有的重视 ${ }^{[4]}$. 直到 1953 年, Beringer 等 ${ }^{[6]}$ 在前人工作的基础上, 报道 了二芳基碘鎓盐与有机碱和无机碱的亲核反应, 发现二 芳基碘鎓盐可以与碳、氢、氧、硫、氮、磷、硒、砷、 锑以及卤素类亲核试剂反应，生成芳基化产物. 对此, 他们认为二芳基碘鈆盐有望成为一种普适性的芳基化 试剂. 后来的研究证实了 Beringer 的预测，二芳基碘鎓 盐在偶联反应，羰基 $\alpha$ 位芳基化反应以及对杂原子的芳 基化反应等方面得到了广泛的应用. Merritt 和 Olofsson $^{[7]}$ 在 2009 年对二芳基碘鎓盐进行了详细的综 述，然而近年来对二芳基碘鈆盐研究的深入，在对其制 备方法的改进、新反应类型的发现以及反应机理的阐释 等方面都取得了很多代表性的成果. 本文从反应发展的 角度出发，综述了二芳基碘鎓盐的结构和反应特性,

\footnotetext{
*E-mail: xiachengfeng@mail.kib.ac.cn

Received March 26, 2013; revised April 28, 2013; published online May 9, 2013.

Project supported by the National Natural Science Foundation of China (No. 21072201) and the National Basic Research Program of China (973 Program, No. 2011CB915500).

国家自然科学基金(No. 21072201)和国家重点基础研究发展计划(973 计划, No. 2011CB915500)资助项目.
} 
2009 年以来取得的新进展以及反应特点、机理和应用 等

\section{1 结构和活性}

二芳基碘鎓盐是一种沿用至今的叫法，国际纯粹与 应用化学联合会(IUPAC)将它命名为 “二芳基- $\lambda^{3}$-碘烷” (diaryl- $\lambda^{3}$-iodanes), 其中, $\lambda^{3}$ 表示碘原子为三价态. 事实 上, “鎓盐” 的叫法有一定的误导性, X 单晶衍射研究表 明，二芳基碘鎓盐并不是以离子对形式存在的盐，而是 一种 $\mathrm{T}$-型结构的分子 ${ }^{[8]}$. 从三维空间来看是一个三角锥 型(图 2a): 碘原子处在中心, 芳基和两对孤对电子平均 分布在平面上, 两个配体垂直于平面分布. 但是两对孤 对电子并没有成键, 因此只是一个假想的三角雉型. 二 芳基碘鎓盐在溶液中的结构仍有争议, 主要受平衡阴离 子 $\left(\mathrm{X}^{-}\right)$和溶剂的影响 ${ }^{[8]}$. (a)

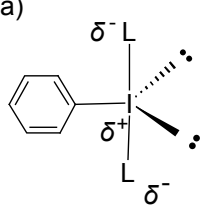

(b)

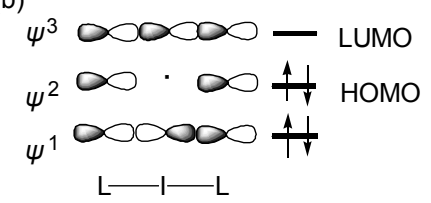

图 2 (a) $\lambda^{3}$-碘烷的结构和(b)高价键的分子轨道

Figure 2 (a) Structure of $\lambda^{3}$-iodane, and (b) molecular orbitals of the hypervalent bond

二芳基碘鎓盐在反应时通常表现为亲电性. 从分子 轨道来看(图 2b), 在它的 HOMO 非成键轨道上有一节 点, 电子云更多的分布到它的两个配体上, 这使得碘原 子成为一个弱的亲电中心, 可与亲核试剂发生亲核反应 (Scheme 1a). 此外，二芳基碘鎓盐还可以被过渡金属活 化，生成高亲电性的金属芳基化物. 金属芳基化物既可 以与芳烃类发生偶联反应还可以与相对 “软” 的亲核试 剂反应(Scheme 1b).

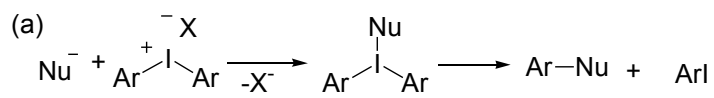

(b)

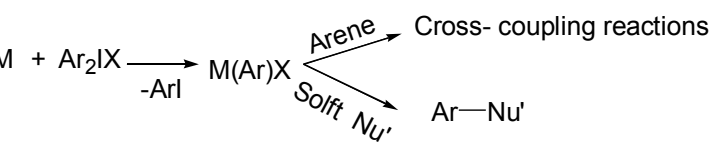

\section{Scheme 1}

二芳基碘鎓盐的溶解性和反应性能受平衡阴离子 $\left(\mathrm{X}^{-}\right)$的影响. 当阴离子为卤素时, 碘鎓盐在大部分有机 溶剂中溶解性差. 此外, 由于卤素负离子本身弱的亲核 性, 可能导致一些副反应的发生. 在应用中, 较多地选 用三氟甲磺酸负离子、四氟硼酸负离子等非亲核性阴离 子作为平衡阴离子. 但是, 也有以卤素作为平衡阴离子
反应进行很好的例子 ${ }^{[9]}$.

二芳基碘鎓盐的亲电性能和平衡阴离子的具体影 响将在下面实例中讨论和分析.

\section{2 制备方法}

自从二芳基碘鎓盐首次被合成以来，后续发展出来 的制备方法已有数十种. 不同方法之间的区别在于使用 不同的氧化试剂、酸以及金属试剂等 ${ }^{[7]}$. 二芳基碘鎓盐 的制备一般需要两至三步反应：第一步是制备三价碘中 间体，可将芳基碘化物氧化生成三价碘，或者是直接用 市售的的三价碘. 接下来这一步根据反应机理的不同, 可大致分为 2 种: 跟富电子芳烃的芳香族亲电取代反应 (Scheme 2a) 和与金属试剂的金属一碘交换反应(Scheme $2 b)$. 这两步也可以不需要分离, 在一锅内完成. 制得的 二芳基碘鎓盐可能还需要一步阴离子交换反应，才能得 到特定的平衡阴离子. 具体的制备方法在之前的综述 ${ }^{[7]}$ 中已有详尽的总结, 这里不再复述. 下面将重点介绍广 泛应用的 “一锅法” (one-pot)和区域选择性制备二芳基 碘鎓盐的方法，最近取得的新进展以及制备手性二芳基 碘鎓盐的一些尝试.

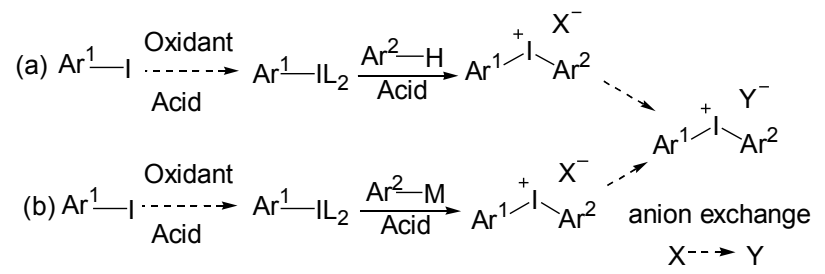

\section{Scheme 2}

目前，广泛应用的是 Olofsson 课题组 ${ }^{[10]}$ 发展的 “一 锅法” 合成: 以碘代芳烃 5 和芳烃 6 为底物，在三氟甲 磺酸作用下，用 $m$-CPBA 作氧化剂，在室温下反应(Eq. 1)数小时内就能完成. 该方法有着良好的底物适应性, 反应条件温和，产率较高(51\% 92\%)，后处理简单。如 果要得到对称的二芳基碘鎓盐 $\left(\mathrm{R}^{1}=\mathrm{R}^{2}\right)^{[11]}$, 可直接用芳 烃类为原料，加入单质碘一锅反应即可.

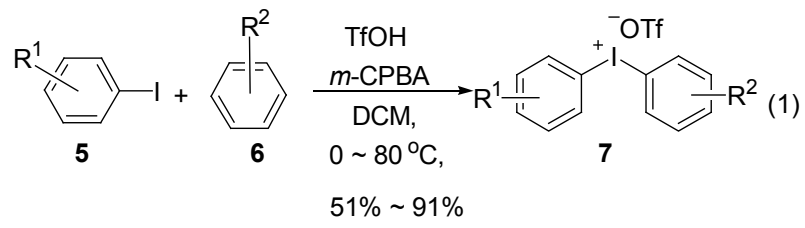

$\mathrm{R}^{1}=\mathrm{H}, 4-\mathrm{Br}, 4-\mathrm{Me}, 4-t-\mathrm{Bu}, 4-\mathrm{NO}_{2}, 4-\mathrm{CF}_{3}, 2-\mathrm{Me}, 4-\mathrm{HOOC}, 3-\mathrm{CF}_{3}$, $\mathrm{R}^{2}=\mathrm{H}, 4-\mathrm{Br}, 4-\mathrm{Me}$, 4-t-Bu, 4-Cl, 4-MeO, 4-F, 2,4,6- $\mathrm{Me}_{3}$, $2,5-\mathrm{Me}_{2}, 2,6-t-\mathrm{Bu}_{2}$

然而，上述方法对于区域选择性的制备芳环上取代 的碘鎓盐仍有很大的局限性，这是由芳烃上取代基的定 
位效应和位阻效应所决定的. 从反应的机理来看: 三价 碘试剂中间体同芳烃反应，是一个芳香族亲电取代过程 (Electrophilic aromatic substitution). 一般给电子基取代 有利于反应进行, 但是给电子基通常有邻位和对位两种 可能，区域选择性差. 吸电子基取代区域选择性较好, 但因反应活性低而使反应进行受阻(Scheme 3). 所以, 用 Olofsson 课题组的方法制备二芳基碘鎓盐常用的策 略是: 选择相对缺电子的芳基碘化物和相对富电子的芳 烃作为底物.

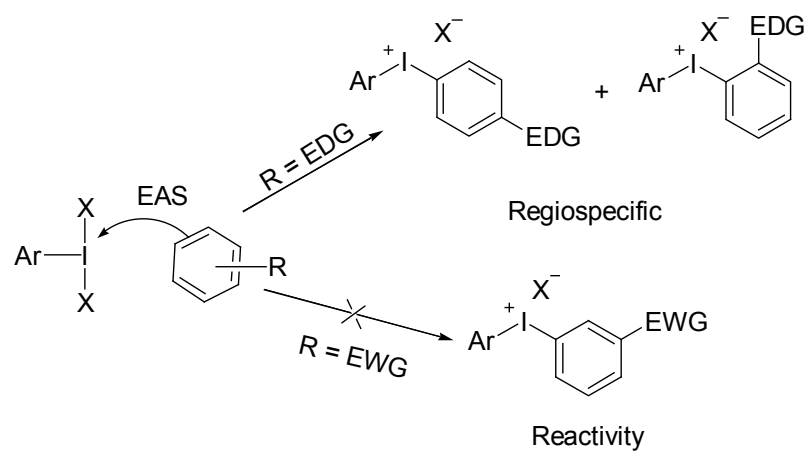

Scheme 3

Koser 等 ${ }^{[12]}$ 用芳基硅烷和羟基甲苯磺酰碘苯(Koser 试剂, HTIB)最早实现了区域选择性的二芳基碘鎓盐合 成. 后来陆续发现芳基三丁基锡 ${ }^{[13]}$ 和芳基硓酸 ${ }^{[14]}$ 都能 实现类似转化，其中芳基硼酸反应温和且产率高，应用 广泛. 这些方法的特点是在硅、锡和硼取代的同位生成 $\mathrm{C}$ - I 键, 且芳环上取代基的影响较小. 但是该方法不足 之处的是需要用事先制备好的三价碘作为底物. 对此, Olofsson 课题组 ${ }^{[15]}$ 发展了 “一锅法” 区域选择性合成二 芳基碘鎓盐的方法(Eq. 2). 只需以碘代芳烃 8 为原料, 在三氟化嗍乙醚存在下经 $m-\mathrm{CPBA}$ 氧化, 再与芳基硼酸 9 反应，实现非对称二芳基碘鎓盐 $\left(\mathrm{R}^{1} \neq \mathrm{R}^{2}\right) \mathbf{1 0}$ 高效合成. 该反应对于制备不同取代基的非对称二芳基碘鎓盐有 广泛的适应性.

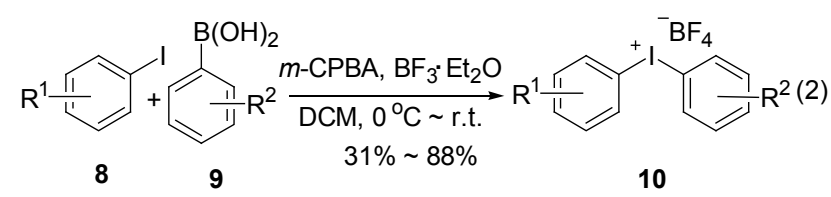

$$
\begin{aligned}
\mathrm{R}^{1}= & \mathrm{H}, 2-\mathrm{F}, 4-\mathrm{Br}, 2-\mathrm{Me}, 3-\mathrm{CF}_{3}, 4-\mathrm{CF}_{3}, 4-\mathrm{MeO}, 4-\mathrm{HCO} \\
\mathrm{R}^{2}= & \mathrm{H}, 2-\mathrm{F}, 4-\mathrm{F}, 3-\mathrm{Br}, 4-\mathrm{Br}, 4-\mathrm{I}, 2-\mathrm{Me}, 3-\mathrm{Me}, 4-\mathrm{t}-\mathrm{Bu}, 4-\mathrm{NO}_{2}, 4-\mathrm{CF}_{3}, \\
& \text { 2,6- } \mathrm{Me}_{2}, 3-\mathrm{CF}_{3}, 4-\mathrm{CF}_{3}, 4-\mathrm{HCO}, 4-\mathrm{MeO}, 3-\mathrm{HCO}-4-\mathrm{MeO}
\end{aligned}
$$

最近, Ermert 等 ${ }^{[16]}$ 为了合成得到对位取代的氟 $\left({ }^{18} \mathrm{~F}\right)$ 苯, 报道了 4-碘代苯基芳基碘鎓盐的简洁合成方法: 先 用 1,4-二碘苯与邻甲基 Koser 试剂进行配体交换得到 4碘代 Koser 试剂, 再在三氟乙醇下 ${ }^{[17]}$ 与芳基嗍酸反应.
Pike 等 ${ }^{[18]}$ 发展了区域选择性二芳基对甲苯磺酸碘鎓盐 的合成方法(Scheme 4). 以碘苯为原料, 先原位合成各 种 Koser 试剂的衍生物 11, 然后同芳基三丁基锡试剂 $\mathbf{1 2}$ 或者是富电子芳烃 13 反应，得到非对称的二芳基碘鎓 盐(14 和 15). Hirschberg 等 ${ }^{[19]}$ 用二芳基四氟硼酸碘鎓盐 与 $N, N$-二(三氟甲基化)铷进行阴离子交换反应，得到 $N, N-$ 二(三氟甲基化)阴离子对的碘鎓盐.

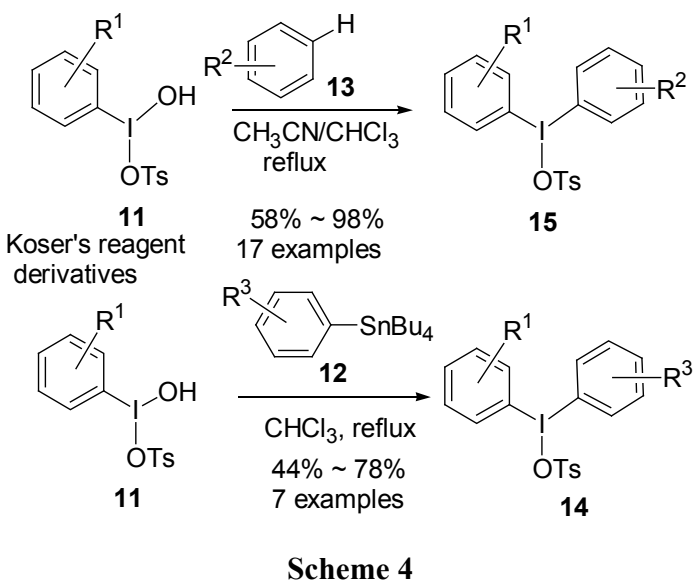

多官能团化尤其是含敏感官能团的二芳基碘鎓盐 的制备一直是个难点. 2012 年, Kita 课题组 ${ }^{[20]}$ 在这方面 取得了突破，报道了含硼取代碘鎓盐的合成方法 (Scheme 5): 嗍取代芳烃 16 与 Koser 试剂(HTIB)发生亲 电取代得到保留硼的碘鎓盐 17 ; 也可以用双硼化芳环 18 与三氟醋酸碘苯(PIFA)反应，选择性地硼碘交换得到 单取代硼的碘鎓盐 17. 该方法一方面巧妙地避开了硼 取代芳烃的副反应，另一方面又利用了不同硼官能团的 活性差别，实现了嗍代碘鎓盐的高效合成.

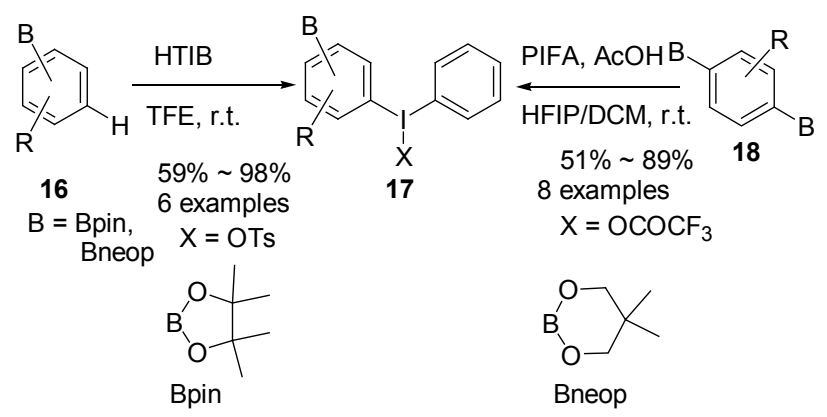

Scheme 5

不对称芳基化反应是有机合成中极富挑战性的难 题，而手性二芳基碘鎓盐有可能发展为不对称芳基化试 剂. Ochiai 等 ${ }^{[21]}$ 最早尝试了这一策略, 以手性联萗作为 骨架，合成出手性碘鎓盐，对映选择性一般. Olofsson 课 题组 ${ }^{[22]}$ 最近发展了基于手性苯酚醚类的碘鎓盐合成方 法，但其应用效果未见报道.

随着二芳基碘鎓盐合成方法的成熟与完善，用它作 
为一种温和的芳基化试剂越来越受到青睐, 由它发展出 来的新转化新反应也层出不穷.

\section{3 二芳基碘鎓盐参与的反应}

二芳基碘鎓盐由于其独特的反应性能，在过渡金属 催化的 $\mathrm{C}-\mathrm{H}$ 键活化/芳基化反应、不含金属的 $\mathrm{C}-\mathrm{H}$ 键 活化/芳基化反应、羰基化合物 $\alpha$ 位芳基化、不对称的串 联环化和芳基化杂原子亲核试剂等方面都得到了广泛 的应用. 尤其是在 $\mathrm{C}-\mathrm{H}$ 活化、不对称芳基化等领域取 得了一系列突破性进展.

\section{1 过渡金属催化的 $\mathrm{C}-\mathrm{H}$ 芳基化反应}

钯催化的偶联反应已经广泛用于有机合成之中，其 中多数转化(如 Stille, Suzuki-Miyaura, Sonogashira, Hiyama, Kumada, Negishi 反应)都需要卤代芳烃与一个活 化的有机金属试剂反应，这在一定程度上限制了它们的 应用. Sanford 课题组 ${ }^{[23]}$ 首先报道了以二芳基碘鎓盐作 为芳基化试剂, 不需要使用活化有机金属试剂的 $\mathrm{C}-\mathrm{H}$ 活化/芳基化反应. 在醋酸钯催化下，二芳基碘鎓盐与芳 烃或含氮杂原子芳环 19 反应得到偶联产物 20 (Scheme $6 a)$. 如果用传统的碘苯或苯酚三氟甲磺酸酯替代二芳 基碘鎓盐，反应几乎不进行. 同年, Daugulis 等 ${ }^{[24]}$ 报道 了邻位 $\mathrm{C}-\mathrm{H}$ 芳基化苯胺 21 的反应, 反应得到邻位二芳 基取代的偶联产物 22 (Scheme 6b).

(a)<smiles>Cc1cccnc1-c1ccccc1</smiles>

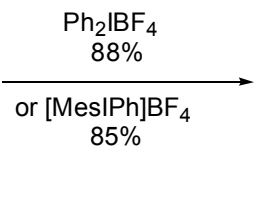

19

(b)

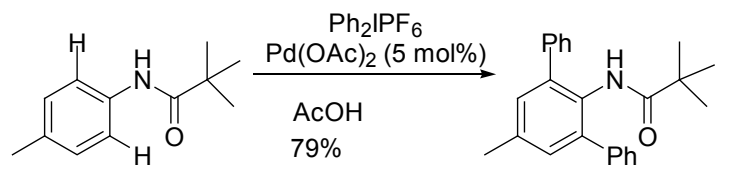

21

Scheme 6

Sanford 研究了非对称二芳基碘鎓盐反应时的区域 选择性, 结果表明位阻小的芳基迁移能力更强, 因而选 择性地参与偶联反应. 他们选用均三甲苯(Mes)作为位 阻化芳基，实现了一系列非对称二芳基碘鎓盐的区域选 择性反应，且产率基本不变(Scheme 6a).

Sanford 课题组 ${ }^{[25]}$ 对这类 $\operatorname{Pd}(\mathrm{II})$ 催化二芳基碘鎓盐 的 C-H 芳基化反应的机理作了深入的研究. 研究表明反 应经历 $\mathrm{Pd}(\mathrm{II}) / \mathrm{Pd}(\mathrm{IV})$ 的催化循环: Pd(II)催化剂以二聚体 的形式 23 参与催化过程，二芳基碘鎓盐先对 23 氧化加 成, 生成 $\operatorname{Pd}(\mathrm{IV}) / \mathrm{Pd}(\mathrm{II})$ 二聚体 24, 紧接着 24 还原消除得 到 25, 底物 19 与 25 配体交换后得到产物 20 (Scheme 7).
其中，二芳基碘鎓盐对 $\operatorname{Pd}(\mathrm{II})$ 加成生成高氧化态的 $\operatorname{Pd}(\mathrm{IV}) / \mathrm{Pd}(\mathrm{II})$ 二聚体是反应的决速步骤.

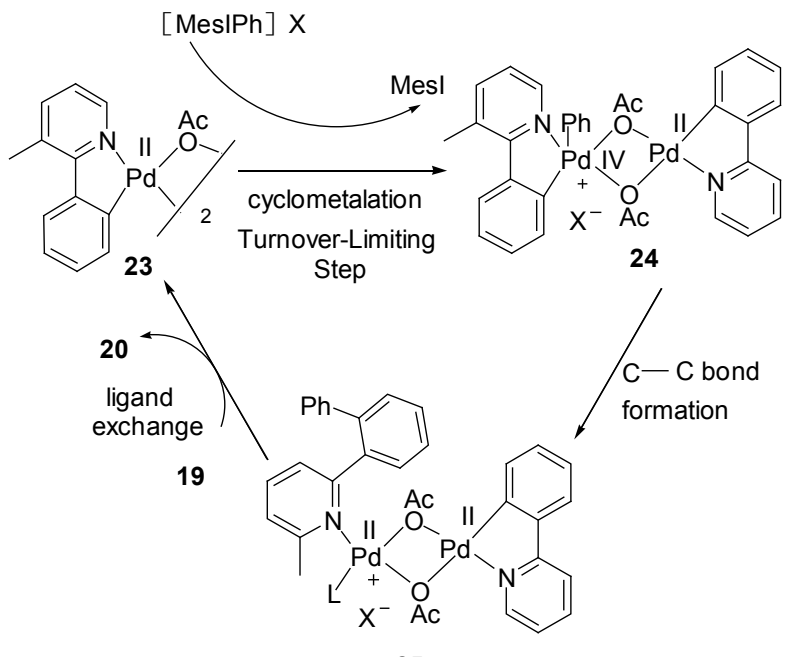

25

\section{Scheme 7}

Gaunt 课题组 ${ }^{[26]}$ 报道了用铜催化、二芳基碘鎓盐参 与的一系列 $\mathrm{C}-\mathrm{H}$ 芳基化反应. 其中, 以吲哚为底物: 1位不保护时得到的是 3-位芳基化的吲哚; 若用乙酰基保 护吲哚 1-位，同样反应条件下得到的是 2-位芳基化产物 (Scheme 8, 28). 其结果与 Sanford 等 ${ }^{[27]}$ 发展的钯催化表 现出完全不同的选择性：二芳基碘鎓盐在 $\operatorname{Pd}(\mathrm{II})$ 催化下, 反应只选择性 $\mathrm{C}-\mathrm{H}$ 芳基化吲哚 3-位(Scheme 8, 26).

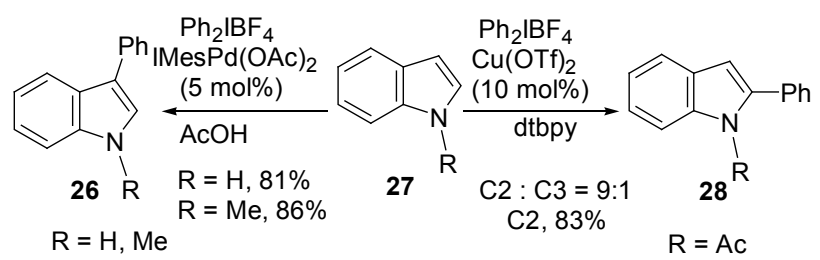

\section{Scheme 8}

Gaunt 课题组 ${ }^{[26 b]}$ 以芳香酰胺为底物, 在铜催化下, 得到的是间位芳基化偶联产物(Eq. 3). 而类似底物，钯 催化下得到的是邻对位产物 ${ }^{[24]}$. 反应有悖于经典的富 电子芳烃邻对位选择性，出乎意料地实现了间位选择 性，这是二芳基碘鎓盐化学取得的重要突破。

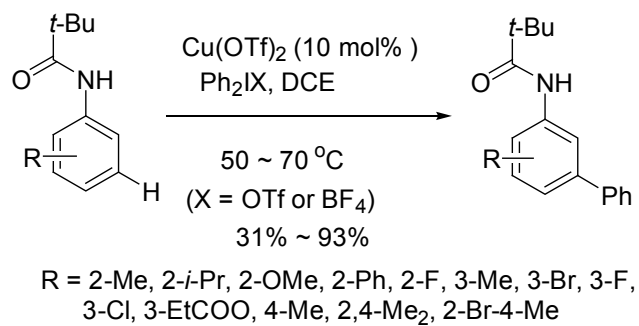

这一非传统的转化, 其反应机理也可能是极其新颖 的. Gaunt 等 ${ }^{[26 b]}$ 提出了芳香酰胺氧参与的三价芳基铜活 
化芳环间位氢的可能机理, 但是并没有进行实验上的验 证. 对此, $\mathrm{Wu}$ 和 $\mathrm{Li}$ 课题组 ${ }^{[28]}$ 进行了密度泛函理论计算 和实验研究. 研究表明, 反应需要经历由活性三价芳基 铜组成的类似 Heck 反应的四元环过渡态 (Scheme 9). 当 间位有甲氧基取代时, 反应还存在一种竞争性的芳环亲 电取代 $\left(\mathrm{S}_{\mathrm{E}} \mathrm{Ar}\right)$ 机理. 前者得到间位偶联产物, 后者得到 邻位偶联产物. 这解释了 Gaunt 结果中 3 位甲氧基取代 底物得到 2 位偶联产物这一意外结果. 但是, 反应机理 中活性三价芳基铜中间体 ${ }^{[29]}$ 的来源仍不能解释. Lockhart ${ }^{[30]}$ 最早研究了铜盐催化二芳基碘鎓盐的反应机理, 认为高亲电性的三价芳基铜是这类反应的活性中间体, 且三价芳基铜是由一价铜氧化而来. 然而, 反应中 Gaunt 加入的催化剂是二价铜. $\mathrm{Wu}$ 等的结果表明, 反应 中真正催化反应的不是加入的二价铜, 而是原位生成的 一价铜.

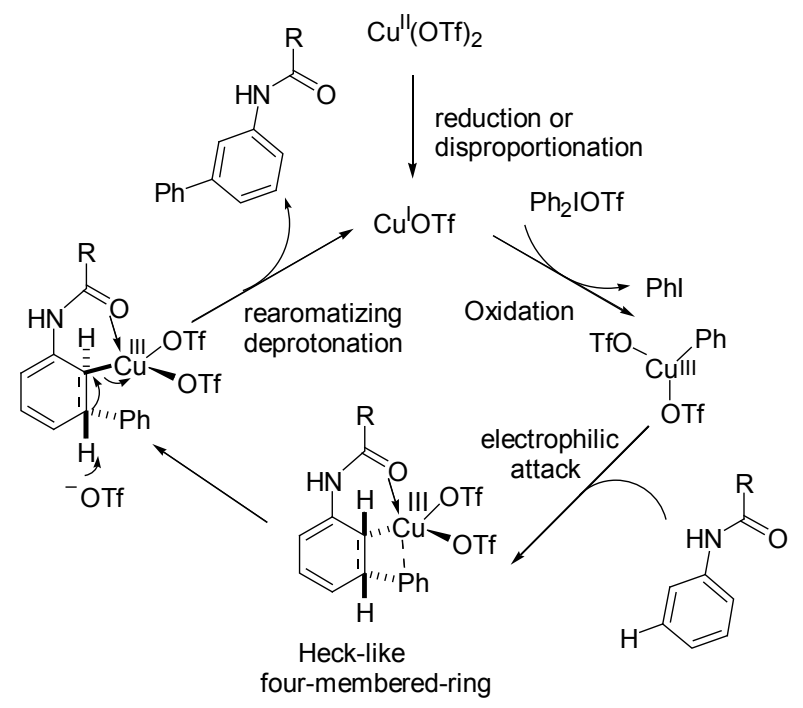

Scheme 9

Gaunt 课题组 ${ }^{[31]}$ 又用 $\alpha$ 位羰基化的芳基化合物作为 底物, 反应选择性地得到间位芳基化产物(Scheme 10a). 在该反应条件下, 羰基 $\alpha$ 位可能的亲核性副反应得到了 有效的控制.

他们课题组 ${ }^{[33]}$ 又报道了苯胺或苯酚类底物的对位 选择性芳基化反应(Scheme $10 \mathrm{~b}$ 和 10c $)^{[32]}$. 即使底物是 游离苯酚类, 主产物仍是二联苯类产物, 而不是二芳基 醚类. 这一系列反应良好的区域选择性, 在苯胺 29 多重 芳基化时得到了良好的体现：反应选择性在苯胺邻、间 和对位上分别引入不同的芳基, 以高产率得到 $\mathbf{3 0}$ (Scheme 11).

Greaney 等 ${ }^{\left[{ }^{[3]}\right.}$ 报道了 Herrmann-Beller 钯催化剂催化 的二芳基碘鎓盐与非活化芳烃的 $\mathrm{C}-\mathrm{H}$ 芳基化反应(Eq. 4). 反应加入三氟乙酸, 增强钯催化剂的亲电性, 是反

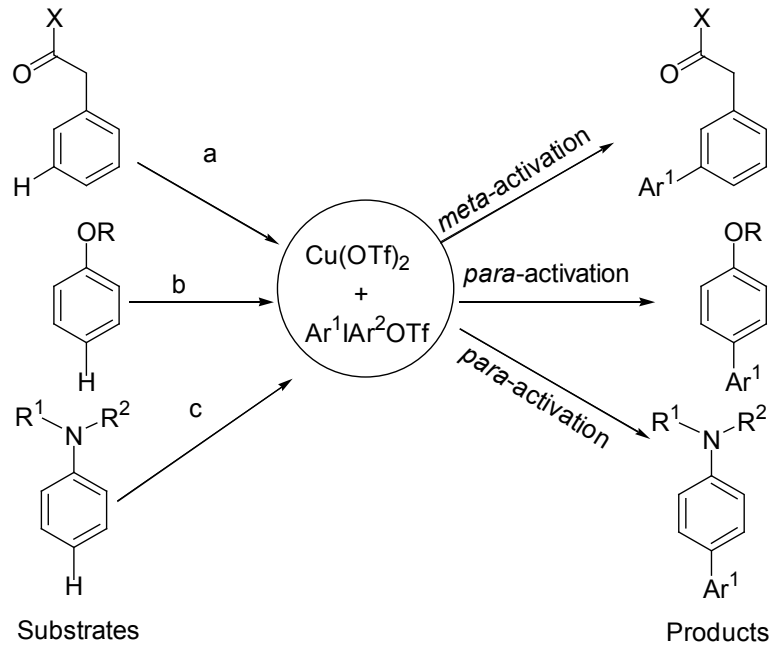

Scheme 10

应成功的关键. 他们将该反应用于具有免疫抑制活性的 多取代联苯类化合物合成. 仅用两步反应，以高达 $93 \%$ 的总产率实现了该化合物的合成.

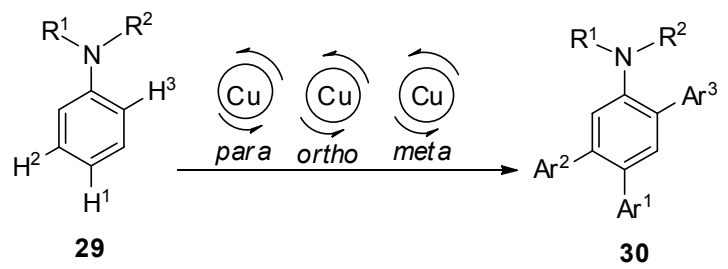

Scheme 11

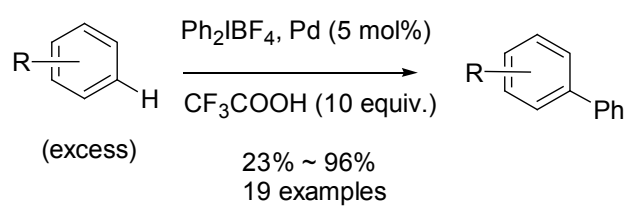

上述工作都是以芳烃作为碘鎓盐的亲核试剂，得到 的是联苯类偶联产物. 2012 年, Gaunt 等 ${ }^{[35]}$ 报道了在铜催 化下，烯烃与二芳基碘鎓盐的类 Heck 偶联反应(Scheme 12). 反应底物适用范围广, 大部分底物以中等偏上的 产率完成反应. 他们提出反应可能经由碳正离子的反应 机理.

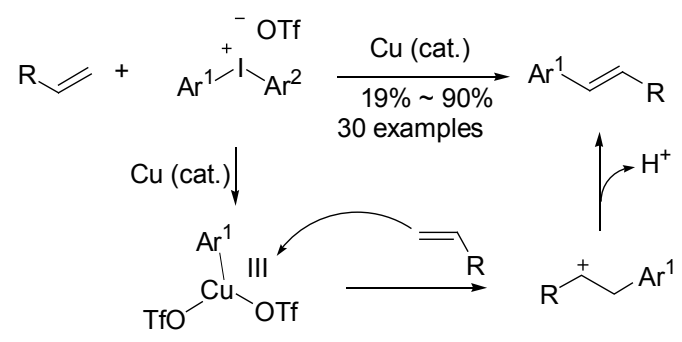

Scheme 12

值得注意的是, 他们成功地将该反应用于新型的串 联反应中. 一个是芳基化-半频哪醇(semi-pinacol)重排 
反应：以烯丙醇 31 为底物, 在标准反应条件下, 反应以 71\%的产率得到重排的芳基化产物 32 (Scheme 13a). 另 一个是芳基化-Grob 碎裂化反应: 以降冰片醇 33 为底物, 在该反应条件下, 以 $61 \%$ 的产率得到碎裂化产物 34 (Scheme 13b). 这些实验结果也初步验证了他们在机理 上提出的假设.

(a)

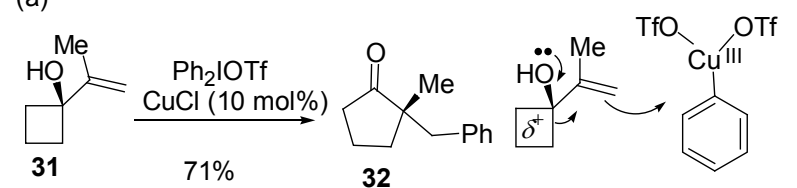

(b)

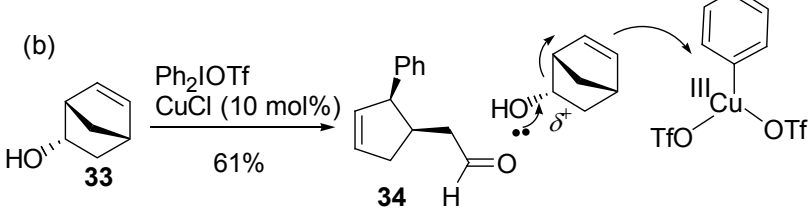

Scheme 13

2013 年, Gillaizeau 等 ${ }^{[36]}$ 报道了以烯胺 35 为底物, $\mathrm{Cu}(\mathrm{OTf})_{2}$ 催化与二芳基碘鎓盐的偶联反应, 得到 3-位芳 基化烯胺产物 36 (Eq. 5). 该方法不足的是需要使用 2 equiv.的二芳基碘鎓盐.

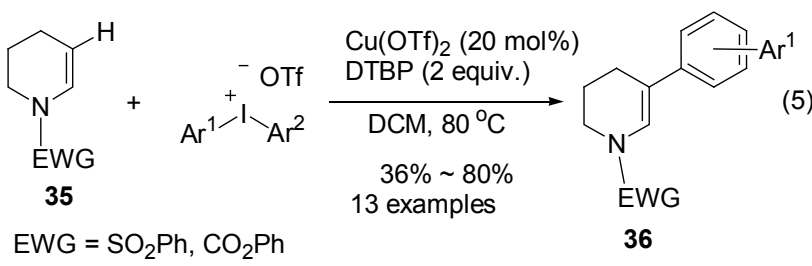

$\mathrm{Li}$ 等 ${ }^{[37]}$ 报道了醋酸钯催化二芳基碘鎓盐对丙烯酸 酯类化合物的 Heck 反应. Varma 等 ${ }^{[38]}$ 报道了二芳基碘鎓 盐在可回收利用的钯催化剂催化下与烯烃的偶联反应, 反应在超声条件下几分钟内即完成.

\section{2 不含金属催化剂的偶联反应}

二芳基碘鎓盐因其自身缺电子性质和碘苯部分的 易离去性，即使没有过渡金属的催化，也能作为有效的 芳基化试剂参与反应. Kita 等 ${ }^{[39]}$ 首次报道在不加金属催 化剂的条件下，实现了两个非活化芳烃之间的氧化偶联 反应, 生成 $\gamma$ 位偶联产物(Scheme 14a). 2010 年, Kita 课 题组 ${ }^{[9]}$ 又实现了同位(ipso)的氧化偶联反应：以富电子 芳烃碘鎓盐 $\mathbf{3 7}$ 为底物, 在 TMSOTf 作用下, 与 $\mathbf{3 8}$ 反应 得到富电子偶联产物 39. 这表明非对称二芳基碘鈆盐 在不含金属催化下, 富电子芳基迁移能力强. 机理研究 表明, 37 先与 38 形成电荷转移复合物(charge-transfer complex), 复合物经单电子转移氧化(SET)引发后, 杂芳 基转移到富电子芳烃上得到偶联产物(Scheme 14b).

Ackermann 等 ${ }^{\left[{ }^{40]}\right.}$ 在 2011 年报道了无需金属存在, a)

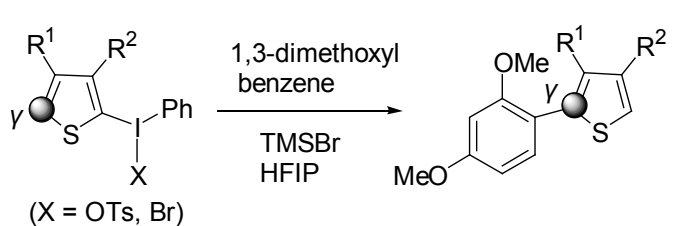

$(\mathrm{X}=\mathrm{OTs}, \mathrm{Br})$

$\mathrm{R}^{1}, \mathrm{R}^{2}=$ alkyl

b)

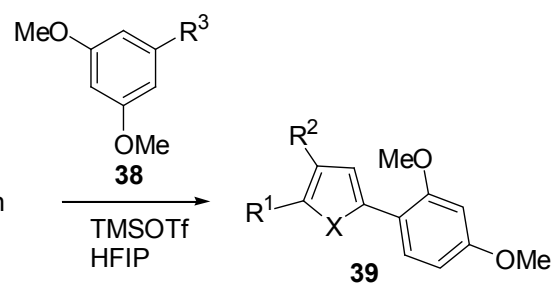

$\mathrm{X}=\mathrm{S}, \mathrm{O}, \mathrm{NAr}$
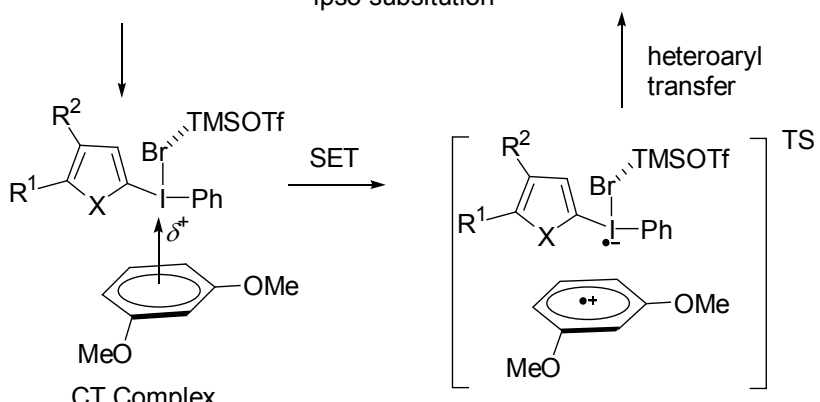

Scheme 14

二芳基碘鎓盐对吲哚和吡咯的偶联反应(Eq. 6). 反应体 系甚至不需要加碱, 只须加热即能进行, 产率中等 (43\% 77\%).

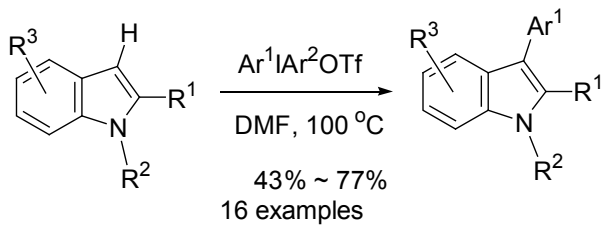

2012 年, $Y u$ 等 ${ }^{[41]}$ 不用金属催化剂, 实现了二芳基碘 鎓盐对芳烃和含氮杂环的芳基化偶联反应，反应只需在 碱性条件下即可进行(Eq. 7). 他们对反应机理进行了初 步研究, 加入自由基抑制剂 TEMPO 后, 反应几乎不进 行; 加入较弱的抑制剂 1,1-二苯基乙烯，反应产率明显 下降，初步证明反应经历的是自由基过程.

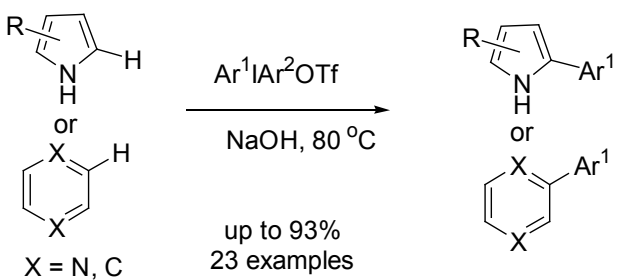

Rrodriguez 等 ${ }^{[42]}$ 于 2012 年报道了对萗的 $\mathrm{C}-\mathrm{H}$ 芳基 化反应(Eq. 8). 与前人报道不同的是，该反应无需过渡 金属催化或碱性条件，以普通芳环荎类作为底物，在微 
波下就能实现. 在等物质的量的自由基捕获剂 TEMPO 存在下, 反应不进行. 在体系中加入各类酸, 也不能促 进产物的生成. 因此, 他们提出自由基过程的反应机理.<smiles>c1ccc2ccccc2c1</smiles>

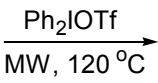

$70 \%(\alpha: \beta=7: 1)$<smiles>c1ccc(-c2cccc3ccccc23)cc1</smiles>

(8)

\section{3 羰基化合物的芳基化}

二芳基碘鎓盐与羰基化合物的反应是另一大类反 应. 这类反应的实质是羰基化合物以烯醇式(包括烯胺、 烯醇硅醚等)对二芳基碘鎓盐的亲核进攻. 最早发现二 芳基碘鎓盐参与的这类反应是同 1,3-二羰基化合物的反 应 $^{[43]}$. 该反应选择性差且产率低, 并没有受到应有的重 视. 直到 2011 年, MacMillan 等 ${ }^{[44]}$ 报道了二芳基碘鎓盐 对醛的不对称芳基化反应，才取得真正的突破. 之后在 这个领域取得了一系列重要进展，下面将一一列举.

MacMillan 课题组 ${ }^{[45]}$ 在这方面的工作是从不对称引 入三氟甲基开始的. 他们采取亚胺结合金属的协同催化 模式, 分别活化醛和三价碘试剂, 以高产率和高对映选 择性完成反应. 协同催化概念的引入以及三价碘试剂的 出现为后面的工作奠定了基础.

他们 ${ }^{[44]}$ 于 2011 年报道了用二芳基碘鎓盐不对称芳 基化醛的反应. 醛 40 在亚胺催化剂 42 催化下, 与溴化 亚铜催化二芳基碘鎓盐 41 反应，得到 $\alpha$ 位芳基的醛 43 . 该反应的产率很高, 对映选择性也非常好. 特别是反应 表现出良好的官能团耐受性, 这是其他方法 ${ }^{[46]}$ 所不具 备的特点. 他们提出了可能的机理解释(Scheme 15).
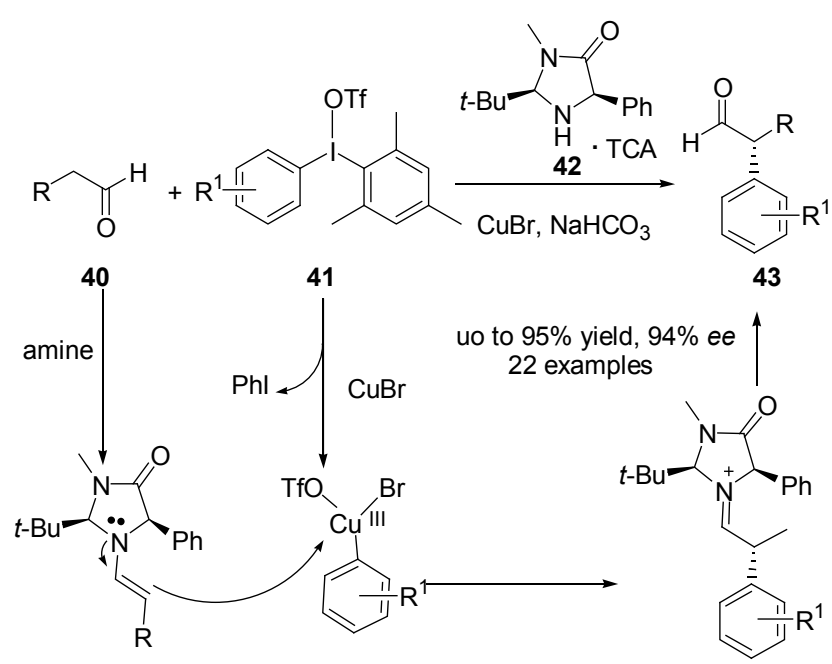

\section{Scheme 15}

他们将该法用于抗炎药酮洛芬的合成, 以市售原料 出发仅用三步就实现了其不对称合成(Eq. 9).

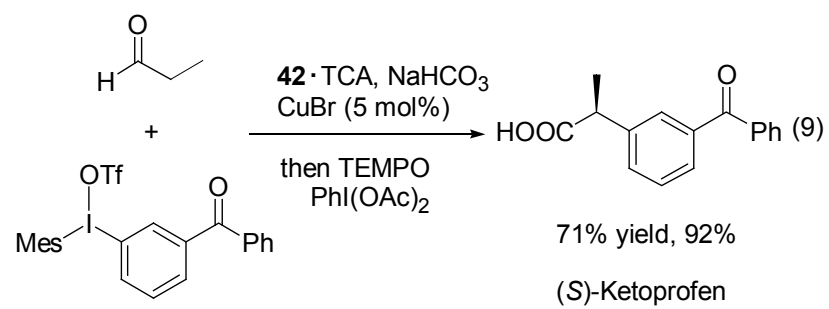

同一年, MacMillan 课题组 ${ }^{[47]}$ 和 Gaunt 课题组 ${ }^{[48]}$ 同 时报道了羰基化合物的不对称芳基化反应(Eq. 10). 在 催化剂 46 的催化下，烯醇硅醚 44 与碘鎓盐 45 反应，生 成 $\alpha$ 位芳基化的羰基产物 47. 两个课题组的方法相似, 在产率和立体选择性都得到了很好的结果.

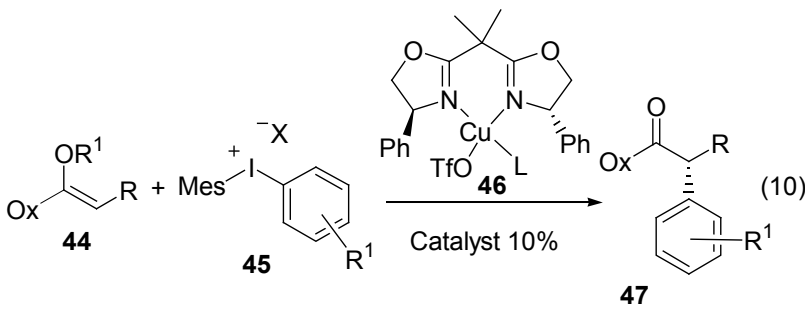

Ox = oxazolidone

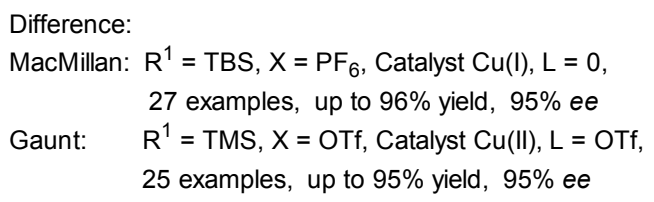

该反应机理与协同催化醛的不对称芳基化反应的 机理不同, 他们提出了可能的机理(Scheme 16).

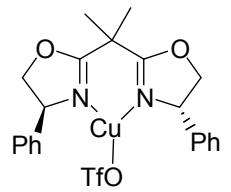<smiles>[R]c1cccc(C([R])C(=O)N2CCOC2=O)c1</smiles><smiles>[R12]c1ccc(I([Y])C)cc1</smiles>

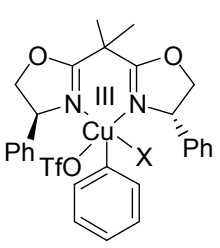<smiles>[R]C=C(O[R17])N1CCOC(=O)C1CC(C)C(=O)O</smiles>

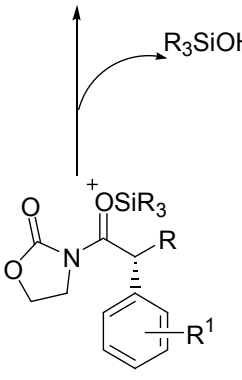

\section{Scheme 16}

Gaunt 课题组将它用抗炎药布洛芬的不对称合成, 反应以克级进行, 产率几乎定量, 目标产物 $e e$ 值达到了 93\% (Eq. 11).

铜催化二芳基碘鎓盐对羰基的不对称芳基化反应， 其实质是 “软” 的亲核试剂(如烯胺，烯醇硅醚等)对铜 活化的二芳基碘鎓盐的亲核反应。还有一种类似的有 


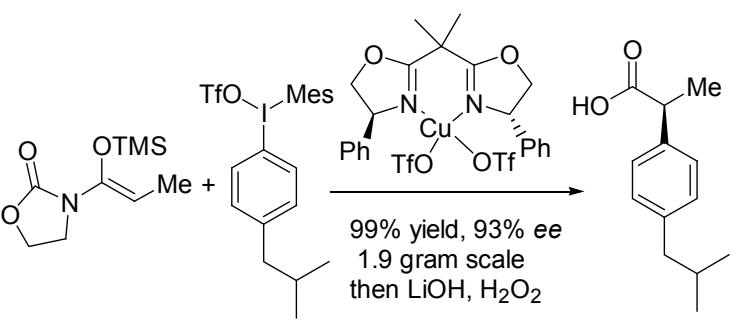

(S)-ibruprofen

机小分子催化-NHC 催化, 其实质也是烯胺进攻, 也 是对羰基化合物的芳基化, 得到的却是芳基酮产物. Gaunt 等 ${ }^{\left[{ }^{49]}\right.}$ 于 2013 年最新报道了杂环醛在 NHC 催化下, 芳基碘鎓盐对醛氢的芳基化反应(Eq. 12). 该反应最大 的亮点是无需金属参与协同催化, 只需 NHC 催化即可. 另外, 生成的二芳基酮结构片段普遍存在于药物分子和 天然产物分子中, 且易转化为其它官能团, 具有潜在的 应用价值.

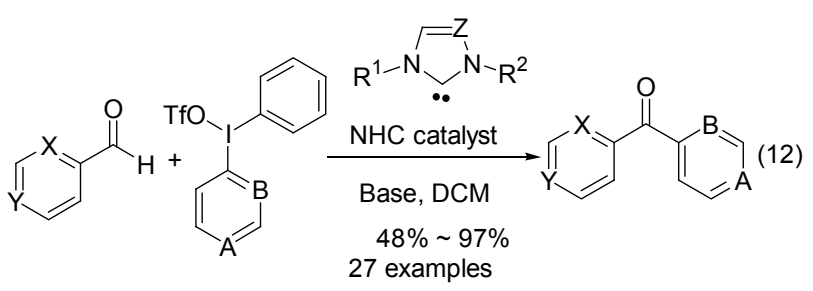

Gaunt 等提出了机理上的假设(Scheme 17).

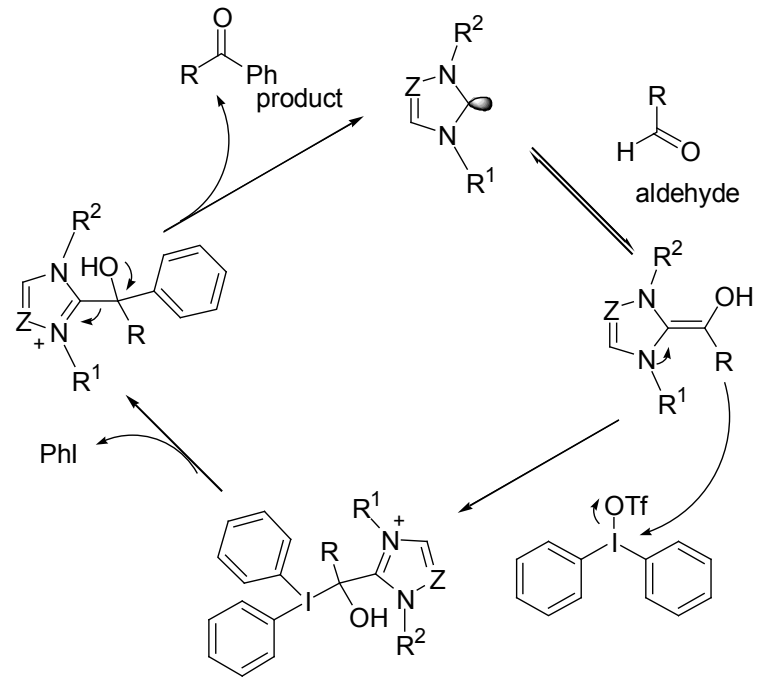

Scheme 17

\section{4 串联环化}

二芳基碘鎓盐作为芳基化试剂，在串联环化反应上 也取得了巨大成功. 2012 年, MacMillan 课题组、Reisman 课题组和 You 课题组相继报道了二芳基碘鈆盐与吲哚 的串联环化反应. 其中, MacMillan 等 ${ }^{[50]}$ 最先报道了吲 哚 48 与碘鎓盐 49 在手性 $\mathrm{Cu}-\mathrm{BOX}$ 催化下的不对称串联 环化反应, 生成吡咯吲哚酮结构 50 (Eq. 13). 反应选用 六氟砷酸作平衡阴离子，有效控制了 2-位芳基化偶联反
应，在 3 位构建了全碳手性季碳. 反应的产率和 $e e$ 值都 非常理想, 吲哚 1 位也可以不需要保护.
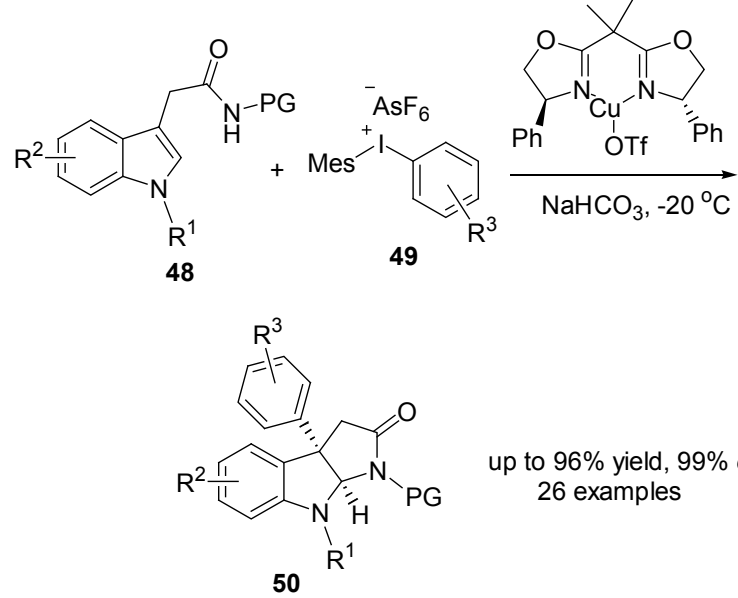

up to $96 \%$ yield, $99 \%$ ee 26 examples

他们提出了可能的反应过程(Scheme 18), 认为反 应过程中生成的亚胺正离子是实现串联环化的关键.

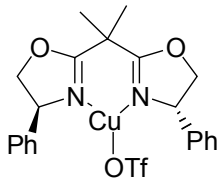

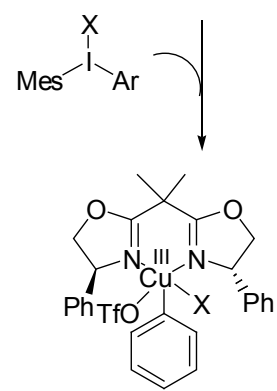

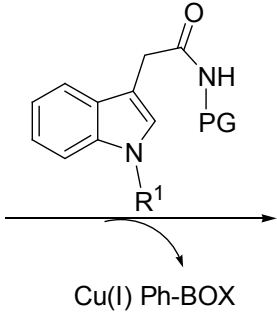<smiles>O=C1C[C@@]2([AlH2])c3ccccc3N([AlH2])[C@@H]2N1P</smiles>

Scheme 18

随后，Reisman 等 ${ }^{[51]}$ 用 Ts 保护的色氨作底物，得到 了类似结果. 反应在非常温和的条件下进行，以中等的 收率得到产物. 反应放大至克级仍能保持在 $63 \%$ 的产 率, 且不需要柱层析. 他们也尝试加入手性配体来实现 不对称转化，但是并没有对映选择性(Eq. 14).

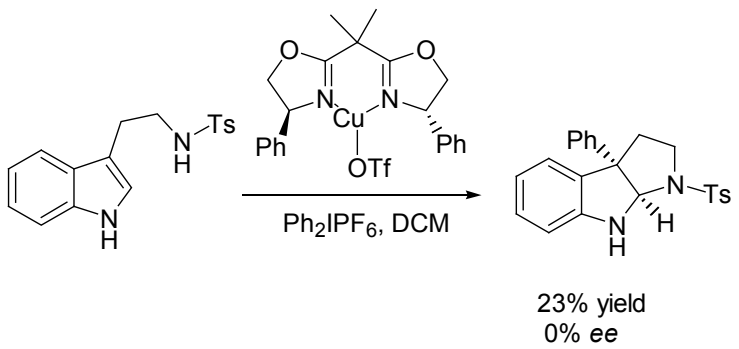

与 Reisman 同时, You 等 ${ }^{[52]}$ 报道了以 2-取代的色胺 醇与二芳基碘鎓盐的串联环化反应，得到呋喃吲哚啉化 
合物(Eq. 15). 反应一步构建了吲哚 2,3-位双季碳中心, 这是极其困难的. 同时, 他们还研究了烯基芳基碘鎓盐 的反应性能, 发现烯基片段也能高效的在 3-位引入.

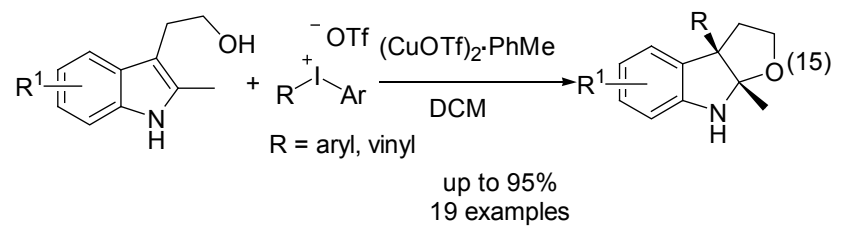

MacMillan 课题组在二芳基碘鎓盐方面的一系列工 作, 随后被杂志《德国应用化学》作为亮点进行了评 ${ }$ 述 ${ }^{[53]}$. 文中认为亲电性的芳基铜 $\mathbf{5 1}$ 是反应的活性中间 体, 将为不对称芳基化反应提供新的途径, 将来可能用 于更多的串联反应中(Scheme 19).

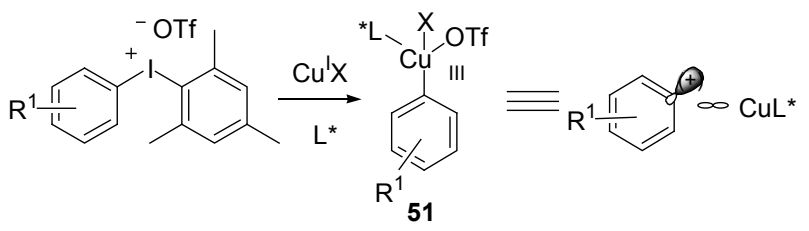

Scheme 19

\section{5 芳基化杂原子亲核试剂}

二芳基碘鎓盐除了偶联反应、与碳亲核试剂的反应 以外, 还可以与各类杂原子发生偶联反应. 其实质是杂 原子亲核试剂或杂原子的孤对电子对二芳基碘鎓盐亲 核进攻. 此部分内容将对 2009 年以后的进展进行综述, 依次介绍 ${ }^{18} \mathrm{~F}$, 氮原子, 氧原子, 硫原子参与的反应.

非天然放射性 ${ }^{18} \mathrm{~F}$ 独特的性能是正极电子成像术 (PET)理想的材料，二芳基碘鎓盐可以用来制备富电子 的 ${ }^{18} \mathrm{~F}$ 代芳烃 ${ }^{[54]}$. 这方面已有专门的综述 ${ }^{[5]}$, 在这里不 再复述. 下面将主要介绍非对称二芳基碘鎓盐与 ${ }^{18} \mathrm{~F}$ 亲 核反应时, 发现的芳基区域选择性迁移现象.

Pike 等 ${ }^{[56]}$ 报道了在微反应器中, 用二芳基碘鎓盐和 ${ }^{18} \mathrm{~F}^{-}$快速高效合成邻位取代的 ${ }^{18} \mathrm{~F}$ 芳烃的方法. 与 Sanford 等 ${ }^{[23]}$ 报道的小位阻芳基选择性迁移不同的是, 氟代时以邻位取代的大位阻基迁移为主(Eq. 16).
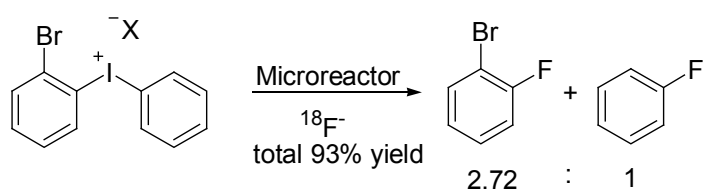

Coenen 等 ${ }^{[57]}$ 发现了 ${ }^{18} \mathrm{~F}$ 与碘鎓盐的亲核反应中另一 种有趣现象: 非对称碘鎓盐 $\mathbf{5 2}$ 参与反应, 可以选择性制 备 ${ }^{18} \mathrm{~F}$ 芳烃 $\mathbf{5 3}$, 而富电子噻吩基部分以碘苯形式离去 (Eq. 17). 说明在该反应中, 缺电子的芳基的迁移能力更 强.

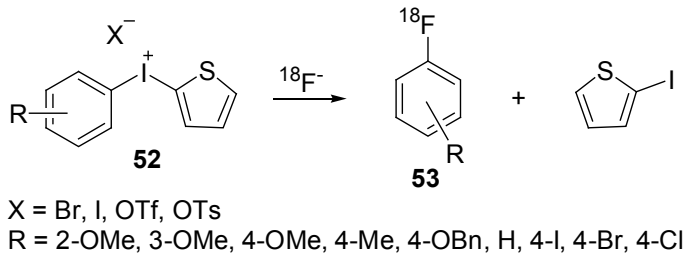

氮原子作为亲核试剂与二芳基碘鎓盐的反应自 2009 年以来已有数例报道. Guo 等 ${ }^{[58}$ 报道了溴化亚铜催 化芳基化嘌啉的反应，反应以高产率实现了嘌啉 9-位的 芳基化. $\mathrm{Li}$ 等 ${ }^{\left[{ }^{[9]}\right.}$ 报道了二芳基碘鎓盐在碱性条件下与液 氨合成芳香胺的反应. Han 等 ${ }^{[00]}$ 发现，二芳基碘鎓盐在 叔丁醇钾存在下，卡巴唑类化合物的 1-位能被芳基化， 反应不需要过渡金属催化, 且条件温和. 如果是用四氢 卡巴唑作底物, 则在吲哚 3-位发生芳基化反应. Varma 等 ${ }^{[6]}$ 报道了砜亚胺类的碳氮偶联反应，反应在超声波 下进行，后处理简单.

Chi 等 ${ }^{[62]}$ 报道了苯乙胺类碘鎓盐 $\mathbf{5 4}$ 碱性条件下,分 子内的亲核关环得到弜哚啉化合物 $\mathbf{5 5}$ (Eq. 18). 反应体 系中加入 0.1 equiv. 的 TEMPO 作为自由基捕获剂, 可有 效抑制碘鈆盐的自由基降解. 反应存在 2 种可能的进攻 方式, 结果表明途径 $\mathrm{a}$ 为主要进攻方式.

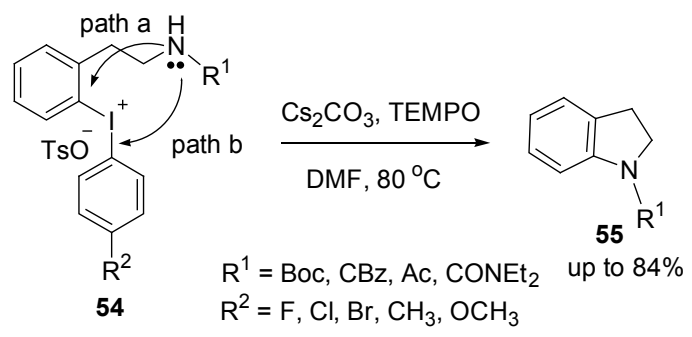

Suna 等 ${ }^{[63]}$ 报道了氮杂环 56 的 $\mathrm{C}-\mathrm{H}$ 活化/叠氮化反 应(Scheme 20). 以氮杂环 $\mathbf{5 6}$ 为原料, 与碘苯二乙酸反 应生成对甲苯磺酰基碘鈆盐 57, 57 在叠氮化钠溶液中发 生配体交换得到 58, 58 在一价铜催化下发生选择性的迁 移得到叠氮化的氮杂环和碘苯, 叠氮官能团在反应体系 中既可以与炔烃反应生成三唑类 $\mathbf{5 9}$, 又可以被硫化铵 还原成胺 60, 两个反应都能以 “一锅法” 实现. 他们对 58 芳基部分的选择性迁移作了机理研究，认为一价铜 催化是实现其选择性迁移的关键.

2011 年, Olofsson 报道 ${ }^{[33]}$ 了苯酚类化合物与碘鎓盐 偶联生成二芳基醚的反应(Eq. 19). 在碱性条件下，反应 只需在室温就能很快完成, 这比以往报道 ${ }^{[6]}$ 的需要剧 烈回流有很大的改进.

该反应有很好的官能团耐受性, 酪氨酸衍生物在该 反应条件下进行氧碳偶联, 产物不会发生消旋化(Eq. 20). 


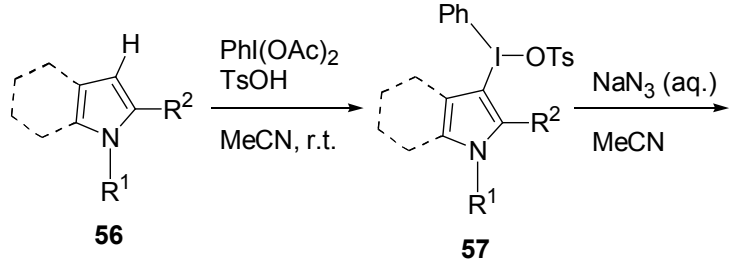

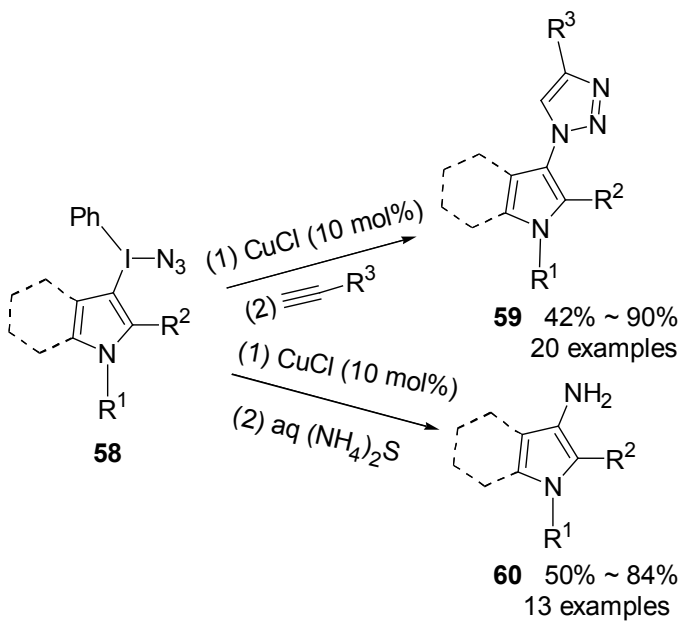

Scheme 20<smiles>[R]c1ccc(Oc2ccc([R])cc2)cc1</smiles>

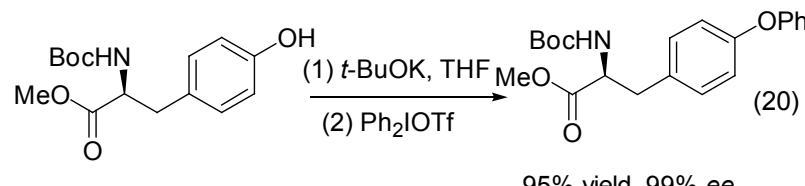

$95 \%$ yield, $99 \%$ ee

随后，他们课题组 ${ }^{[65]}$ 报道了羧酸的芳基化反应(Eq. 21). 反应条件同醚化反应略有不同, 反应需要在甲苯 中回流进行. 芳香羧酸和脂肪羧酸都能以很好的收率完 成反应. 值得关注的是，该反应中二芳基碘鎓盐表现出 不同的区域选择性: 大位阻的 2,4,6-三异丙基苯基团选 择性的生成了羧酸酯.<smiles>[R]C(=O)Oc1c([R])cc([R1])cc1[R]</smiles>

Onomura 等 ${ }^{[66]}$ 报道了以二醇为底物, 铜催化的选择 性芳基化成醚反应(Eq. 22). 在碘鎓盐过量的条件下，反 应能以很好的产率实现单芳基化成醚，他们并没有提到
对映选择性的尝试结果.

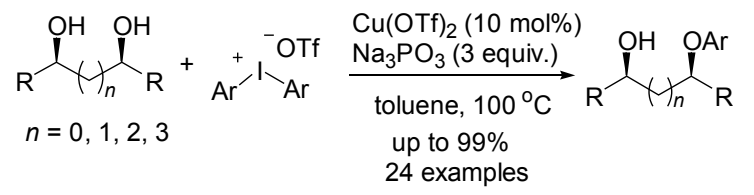

Manolikakes 等 ${ }^{[67]}$ 以芳香亚磺酸钠盐为底物，与二 芳基碘鎓盐偶联生成二芳基砜(Eq. 23). 反应既不需要 加过渡金属催化，也不需要加碱就能高产率的实现转 化. 他们设计实验研究了二芳基碘鎓盐的选择性，发现 反应在铜催化下和无金属催化下表现出完全不同的区 域选择性.

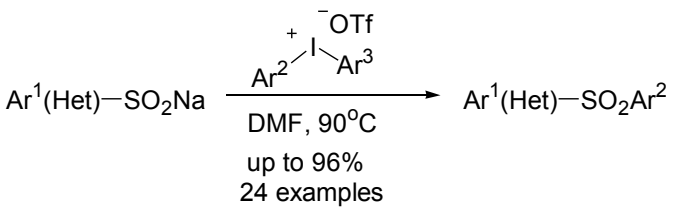

Karade 等 ${ }^{[68]}$ 报道了 61 与碘鎓盐 62 的偶联反应，以 纳米氧化铜为催化剂, 一步实现 $\mathrm{C}-\mathrm{S}$ 键偶联和氧化芳 构化得到 63 (Eq. 24). 纳米氧化铜催化剂可以回收利用, 且催化活性不会下降.

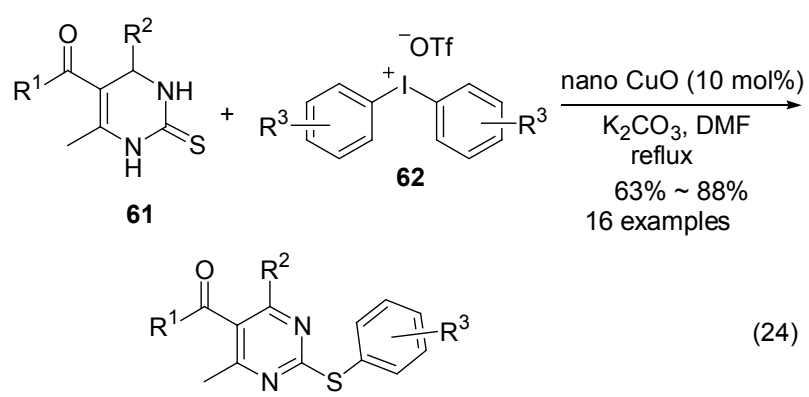

\section{4 总结与展望}

综上所述，二芳基碘鎓盐因其易得、温和、无毒且 性质稳定，在有机合成中作为芳基化试剂得到广泛应 用. 它参与的许多反应，反应条件温和、官能团耐受性 好且区域选择性好. 同时，它可以同多种元素的亲核试 剂反应，是一种普适性的芳基化试剂. 反应既可以在金 属催化下进行，也可以在无金属甚至有机催化条件下进 行. 然而，二芳基碘鎓盐的这些进展大多是最近十年取 得的，仍然有许多问题需要解决. 比如：二芳基碘鎓盐 芳基化的反应机理值得继续探讨 ${ }^{[69]}$ ，在全合成中的应 用需要加大开发 ${ }^{[70], ~}$ 非对称二芳基碘鎓盐反应中的区 域选择性迁移 ${ }^{[71]}$. 另外，二芳基碘鎓盐参与的反应不满 足原子经济性 ${ }^{[72]}$ 的要求：二芳基碘鎓盐反应时只有其 中一个芳基参与反应，另一个芳基生成芳基碘副产物. 
如何实现使用催化量 ${ }^{[73]}$ 的二芳基碘鎓盐作为芳基化试 剂是个难题.

\section{References}

[1] Willgerodt, C. J. Prakt. Chem. 1886, 33, 154.

[2] Willgerodt, C. Ber. 1892, 25, 3494.

[3] Hartmann, C.; Meyer, V. Ber 1893, 26, 1727

[4] Hartmann, C.; Meyer, V. Ber 1894, 27, 426.

[5] (a)Stang, P. J.; Zhdankin, V. V. Chem. Rev. 1996, 96, 1123; (b)Zhdankin, V. V.; Stang, P. J. Chem. Rev. 2002, 102, 2523; (c) Zhdankin, V. V.; Stang, P. J. Chem. Rev. 2008, 108, 5299.

[6] Beringer, F. M.; Brierley, A.; Drexler, M.; Gindler, E. M. Lumpkin, C. C. J. Am. Chem. Soc. 1953, 75, 2708.

[7] Merritt, E. A.; Olofsson, B. Angew. Chem., Int. Ed. 2009, 48, 9052.

[8] Ochiai, M. Top. Curr. Chem. 2003, 224, 5.

[9] Dohi, T.; Ito, M.; Yamaoka, N.; Morimoto, K.; Fujioka, H.; Kita, Y. Angew. Chem., Int. Ed. 2010, 49, 3334.

[10] (a) Bielawski, M.; Olofsson, B. Chem. Commun. 2007, 2521; (b) Bielawski, M.; Zhu, M.; Olofsson, B. Adv. Synth. Catal. 2007, $349,2610$.

[11] Bielawski, M.; Krämer, K.; Olofsson, B. Org. Synth 2009, 86, 308.

[12] Koser, G. F.; Wettach, R. H.; Smith, C. S. J. Org. Chem. 1980, 45, 1543.

[13] W. Pike, V.; Butt, F.; Shah, A.; Widdowson, D. J. Chem. Soc., Perkin Trans. 1 1999, 245.

[14] Ochiai, M.; Toyonari, M.; Sueda, T.; Kitagawa, Y. Tetrahedron Lett. 1996, 37, 8421.

[15] Bielawski, M.; Aili, D.; Olofsson, B. J. Org. Chem. 2008, 73, 4602.

[16] Cardinale, J.; Ermert, J.; Coenen, H. H. Tetrahedron 2012, 68, 4112.

[17] Dohi, T.; Yamaoka, N.; Kita, Y. Tetrahedron 2010, 66, 5775.

[18] Chun, J.-H.; Pike, V. W. J. Org. Chem. 2012, 77, 1931.

[19] Hirschberg, M. E.; Wenda, A.; Frohn, H.-J.; Ignatev, N. V. J. Fluorine Chem. 2012, 138, 24.

[20] Ito, M.; Itani, I.; Toyoda, Y.; Morimoto, K.; Dohi, T.; Kita, Y., Angew. Chem., Int. Ed. 2012, 51, 12555.

[21] Ochiai, M.; Kitagawa, Y.; Takayama, N.; Takaoka, Y.; Shiro, M. J. Am. Chem. Soc. 1999, 121, 9233.

[22] Jalalian, N.; Olofsson, B. Tetrahedron 2010, 66, 5793.

[23] Kalyani, D.; Deprez, N. R.; Desai, L. V.; Sanford, M. S. J. Am. Chem. Soc. 2005, 127, 7330.

[24] Daugulis, O.; Zaitsev, V. G. Angew. Chem., Int. Ed. 2005, 44, 4046.

[25] Deprez, N. R.; Sanford, M. S. J. Am. Chem. Soc. 2009, 131, 11234.

[26] (a) Phipps, R. J.; Grimster, N. P.; Gaunt, M. J. J. Am. Chem. Soc. 2008, 130, 8172;

(b) Phipps, R. J.; Gaunt, M. J. Science 2009, 323, 1593.

[27] Deprez, N. R.; Kalyani, D.; Krause, A.; Sanford, M. S. J. Am. Chem. Soc. 2006, 128, 4972.

[28] Chen, B.; Hou, X.-L.; Li, Y.-X.; Wu, Y.-D. J. Am. Chem. Soc. 2011, 133, 7668.

[29] Casitas, A.; Ribas, X. Chem. Sci. 2013, 4, 2301.

[30] Lockhart, T. P. J. Am. Chem. Soc. 1983, 105, 1940.

[31] Duong, H. A.; Gilligan, R. E.; Cooke, M. L.; Phipps, R. J.; Gaunt, M. J. Angew. Chem., Int. Ed. 2011, 50, 463.

[32] Ciana, C. L.; Phipps, R. J.; Brandt, J. R.; Meyer, F. M.; Gaunt, M. J. Angew. Chem., Int. Ed. 2011, 50, 458.

[33] Jalalian, N.; Ishikawa, E. E.; Silva, L. F.; Olofsson, B. Org. Lett. 2011, 13, 1552.

[34] Storr, T. E.; Greaney, M. F. Org. Lett. 2013, 15, 1410.

[35] Phipps, R. J.; McMurray, L.; Ritter, S.; Duong, H. A.; Gaunt, M. J.
J. Am. Chem. Soc. 2012, 134, 10773.

[36] Gigant, N.; Chausset-Boissarie, L.; Belhomme, M.-C.; Poisson, T.; Pannecoucke, X.; Gillaizeau, I. Org. Lett. 2012, 14, 278.

[37] Li, J.; Liu, L.; Zhou, Y.-Y.; Xu, S.-N. RSC Adv. 2012, 2, 3207.

[38] Vaddula, B. R.; Saha, A.; Leazer, J.; Varma, R. S. Green Chem. 2012, 14, 2133.

[39] Kita, Y.; Morimoto, K.; Ito, M.; Ogawa, C.; Goto, A.; Dohi, T. J. Am. Chem. Soc. 2009, 131, 1668.

[40] Ackermann, L.; Dell'Acqua, M.; Fenner, S.; Vicente, R. N.; Sandmann, R. Org. Lett. 2011, 13, 2358.

[41] Wen, J.; Zhang, R.-Y.; Chen, S.-Y.; Zhang, J.; Yu, X.-Q. J. Org. Chem. 2011, 77, 766.

[42] Castro, S.; Fernandez, J. J.; Vicente, R.; Fananas, F. J.; Rodriguez, F. Chem. Commun. 2012, 48, 9089.

[43] Marshall Beringer, F.; Forgione, P. S.; Yudis, M. D. Tetrahedron 1960, 8,49 .

[44] Allen, A. E.; MacMillan, D. W. C. J. Am. Chem. Soc. 2011, 133, 4260.

[45] Allen, A. E.; MacMillan, D. W. C. J. Am. Chem. Soc. 2010, 132, 4986.

[46] (a) Taylor, A. M.; Altman, R. A.; Buchwald, S. L. J. Am. Chem. Soc. 2009, 131, 9900.

(b) Liao, X.; Weng, Z.; Hartwig, J. F. J. Am. Chem. Soc. 2007, 129, 195 .

(c) Lundin, P. M.; Fu, G. C. J. Am. Chem. Soc. 2010, 132, 11027.

[47] Harvey, J. S.; Simonovich, S. P.; Jamison, C. R.; MacMillan, D. W. J. Am. Chem. Soc. 2011, 133, 13782.

[48] Bigot, A.; Williamson, A. E.; Gaunt, M. J. J. Am. Chem. Soc. 2011, 133, 13778.

[49] Toh, Q. Y.; McNally, A.; Vera, S.; Erdmann, N.; Gaunt, M. J. J. Am. Chem. Soc. 2013, 135, 3772.

[50] Zhu, S.; MacMillan, D. W. C. J. Am. Chem. Soc. 2012, 134, 10815.

[51] Kieffer, M. E.; Chuang, K. V.; Reisman, S. E. Chem. Sci. 2012, 3, 3170.

[52] Liu, C.; Zhang, W.; Dai, L.-X.; You, S.-L. Org. Lett. 2012, 14, 4525.

[53] Rousseaux, S.; Vrancken, E.; Campagne, J.-M. Angew. Chem., Int. Ed. 2012, 51, 10934

[54] Pike, V. W.; Aigbirhio, F. I. J. Chem. Soc., Chem. Commun. 1995, 2215.

[55] Tredwell, M.; Gouverneur, V. Angew. Chem., Int. Ed. 2012, 51, 11426.

[56] Chun, J.-H.; Lu, S.; Lee, Y.-S.; Pike, V. W. J. Org. Chem 2010, 75, 3332.

[57] Ross, T. L.; Ermert, J.; Hocke, C.; Coenen, H. H. J. Am. Chem. Soc. 2007, 129, 8018

[58] Niu, H.-Y.; Xia, C.; Qu, G.-R.; Zhang, Q.; Jiang, Y.; Mao, R.-Z.; Li, D.-Y.; Guo, H.-M. Org. Biomol. Chem. 2011, 9, 5039.

[59] Li, J.; Liu, L. RSC Adv. 2012, 2, 10485.

[60] Guo, F.; Wang, L.; Wang, P.; Yu, J.; Han, J. Asian J. Org. Chem. 2012, 1,218

[61] Vaddula, B.; Leazer, J.; Varma, R. S. Adv. Synth. Catal. 2012, 354, 986.

[62] Landge, K. P.; Jang, K. S.; Lee, S. Y.; Chi, D. Y. J. Org. Chem. 2012, 77, 5705 .

[63] Lubriks, D.; Sokolovs, I.; Suna, E. J. Am. Chem. Soc. 2012, 134, 15436.

[64] Crowder, J. R.; Glover, E. E.; Grundon, M. F.; Kaempfen, H. X. J. Chem. Soc. 1963, 4578.

[65] Petersen, T. B.; Khan, R.; Olofsson, B. Org. Lett. 2011, 13, 3462.

[66] Kuriyama, M.; Hamaguchi, N.; Onomura, O. Chem. Eur. J. 2012, $18,1591$. 
[67] Umierski, N.; Manolikakes, G. Org. Lett. 2012, 14, 188.

[68] Bhong, B. Y.; Shelke, A. V.; Karade, N. N. Tetrahedron Lett. 2012, $54,739$.

[69] Norrby, P.-O.; Petersen, T. B.; Bielawski, M.; Olofsson, B. Chem. Eur. J. 2010, 16, 8251.

[70] (a) Aggarwal, V. K.; Olofsson, B. Angew. Chem., Int. Ed. 2005, 44,
5516 ;

(b) Silva Jr, L. F.; Olofsson, B. Nat. Prod. Rep. 2011, 28, 1722.

[71] Wang, B.; Graskemper, J. W.; Qin, L.; DiMagno, S. G. Angew. Chem., Int. Ed. 2010, 49, 4079.

[72] Trost, B. M. Science 1991, 254, 1471.

[73] Dohi, T.; Kita, Y. Chem. Commun. 2009, 2073. 